\title{
El periodo de Pittsburgh en la vida de Juan Pablo Izquierdo: 1990-2008
}

Juan Pablo Izquierdo, Pittsburgh Period: 1990-2008

\author{
por \\ Marilyn Taft-Thomas, Ph.D. \\ Carnegie Mellon University School, Pittsburgh, Estados Unidos \\ mt3f@andrew.cmu.edu
}

\begin{abstract}
Este artículo describe la carrera profesional de Juan Pablo Izquierdo durante los dieciocho años que se desempeñó como profesor en la Escuela de Música de la Carnegie Mellon University en Pittsburgh, Pennsylvania. Investiga su labor de formar la orquesta estudiantil, el crecimiento del impacto de la escuela mediante proyectos internacionales de radio y de fonogramas, la creación de lazos entre la Escuela y los músicos profesionales jóvenes en Chile, además del desarrollo de las competencias de interpretación y dirección orquestal de los estudiantes que trabajaron con él. La inmensidad de su legado se refleja en las críticas que se publicaron acerca de sus grabaciones del repertorio musical contemporáneo y en los comentarios personales de quienes estudiaron dirección orquestal bajo su guía.

Palabras clave: Juan Pablo Izquierdo, Escuela de Música de la Universidad Carnegie Mellon, Orquesta Filarmónica Carnegie Mellon, música de vanguardia europea y norteamericana del siglo XX, programa avanzado en dirección orquestal, programa de residencia para intérpretes.
\end{abstract}

This article describes the professional career of Juan Pablo Izquierdo during the eighteen years he held a faculty position in the Carnegie Mellon University School of Music, Pittsburgh, Pennsylvania. It traces his work in building the student orchestra, broadening the impact of the school through international radio and recording projects, creating links between the school and young professional musicians in Chile, and developing the performance and conducting skills of the students with whom he worked. The depth of his legacy is reflected in published reviews of his recordings of contemporary repertoire and in personal comments offered by his former conducting students.

Key words: Juan Pablo Izquierdo, Carnegie Mellon University School of Music, Carnegie Mellon Philharmonic Orchestra, European and North American avant-garde music of the twentieth century, advanced degree program in conducting, performance residence program.

Juan Pablo Izquierdo se integró al cuerpo de profesores de la Carnegie Mellon University School of Music en el otoño de 1990 como Director de Estudios Orquestales y se mantuvo en esta posición durante dieciocho años. El maestro Izquierdo cumplió sus responsabilidades en el campus en Pittsburgh, Pennsylvania, con gran pericia y dedicación. Mantuvo simultáneamente su papel como director musical de la Orquesta Sinfónica de Chile y de la Orquesta Claudio Arrau en Santiago, y continuó dirigiendo profesionalmente en grandes ciudades alrededor del mundo.

Durante su permanencia enfocó su obra en el Carnegie Mellon hacia el desarrollo de una orquesta de estudiantes del más alto nivel. Con el apoyo de la 
Dirección de la Escuela, expandió el horario de ensayos de la Carnegie Mellon Philharmonic, trasladó sus presentaciones al Carnegie Music Hall, de excelente nivel acústico, y seleccionó un repertorio que constituía un desafío por la inclusión significativa de obras escritas durante el siglo XX.

Como profesor y director consumados exigía en sus ensayos a cada músico una concentración total y una atención a cada detalle. La afinación se ajustaba a algo cercano a la perfección y esperaba que las interpretaciones fueran tanto de nivel profesional como musicalmente estimulantes. ¡Y así resultaron ser!

El permanente crecimiento de la excelencia de la orquesta atrajo a las audiencias de esta comunidad y finalmente llevó a la realización de presentaciones anuales fuera de la principal región de Pittsburgh. La primera de estas presentaciones se efectuó el 1 de abril de 1995 en el Kennedy Center en Washington, D.C., con la interpretación de música de Edgard Varèse. Al año siguiente, el maestro Izquierdo dirigió a la orquesta juvenil en un programa de música de Iannis Xenakis que se realizó en el afamado Carnegie Hall el 23 de abril de 1996. A este concierto le siguieron otros en los años siguientes con la música de Olivier Messiaen en el Boston's Symphony Hall, Giacinto Scelsi en el Carnegie Hall en Nueva York y un Festival Hindemith en el Severance Hall en Cleveland, Ohio. El rasgo en común que tenían todos estos conciertos fuera del Estado era la presentación de música de vanguardia del siglo XX que era raramente ejecutada. La interpretación imaginativa y aun atrevida de la música contemporánea de Juan Pablo Izquierdo rápidamente le ganó la admiración tanto del público como de los críticos.

Arthur R. Smith, crítico de The Washington Post, describió el concierto del Kennedy Center con estas palabras: "explosiones de sonido decididas, limpiamente interpretadas y maravillosamente controladas...". Continúa diciendo: "En muy pocas obras es tan grande el desafío de mantenerlas integradas. El director Juan Pablo Izquierdo manejó eso y mucho más”. Nota: ni este crítico ni el público supo cuánta verdad encerraban estas palabras.

Un aspecto particularmente desafiante de la interpretación de Amériques de Varèse era el uso de una sirena. El compositor especificó la incorporación de una sirena que pudiera ser interrumpida instantáneamente en ciertos lugares de la partitura. Para realizar la obra tal como había sido concebida por el compositor, una sirena de cincuenta libras de peso se consiguió en un departamento de bomberos de las cercanías. No obstante, existían numerosos problemas técnicos, como la dificultad de interrumpirla, controlar su volumen e impedir que saltara de la mesa. La instalación de la sirena tuvo entonces que ser rediseñada. Se fijó al piso, se agregó un interruptor de dos velocidades para controlar el volumen y se inventó un nuevo freno con un palo de escoba. Todo esto lo logró Ricardo Schulz, Director de Actividades de Grabación. Fue así como el maestro Izquierdo pudo interpretar la composición siguiendo la intención del compositor. Nada menor a esto resultaba aceptable para este notable músico.

El impacto de su enseñanza no se limitó a la Philharmonic Orchestra. En colaboración con Robert Page, profesor de música en Paul Mellon University además de director de ópera y estudios corales en la escuela, Izquierdo desarrolló un programa avanzado conducente al grado de master en dirección de orquesta. 
Siguiendo las huellas de su propio profesor y guía, Hermann Scherchen, el profesor Izquierdo trabajaba intensivamente con cada uno de sus estudiantes privados de dirección orquestal. Insistió siempre en mantener las mismas exigencias para lograr los niveles de excelencia a los que él aspiraba en su propia obra.

Muchos de sus estudiantes siguieron carreras distinguidas en la profesión. Ellos incluyen a las siguientes figuras: Gil Rose, fundador y director artístico de la Boston Modern Orchestra Project (Proyecto de la Moderna Orquesta de Boston), una orquesta profesional de los Estados Unidos comprometida en comisionar, ejecutar y grabar música de los siglos veinte y veintiuno; Nizan Leibovich, director artístico del Israeli Music Days Festival (Festival de los Días Musicales de Israel) en Jerusalén y director de orquestas y conjuntos en los Estados Unidos, Europa e Israel; Andrés Cladera, 2007 Outstanding Young Conductor (Joven Conductor de Excelencia de 2007) de la Asociación de Directores Corales de Norteamérica, y Walter Morales, director de orquesta y pianista, el más importante intérprete de música de Costa Rica en el mundo, quien cuenta con grabaciones realizadas en los sellos Centaur, Mode, Zimbel y Quindecim.

Para Walter Morales, sus estudios con Juan Pablo Izquierdo "cambiaron profundamente su vida". De acuerdo con sus propias palabras, "el maestro Juan Pablo Izquierdo tenía una habilidad misteriosa para desarrollar la capacidad potencial de dirigir en todos sus estudiantes. Todos los días sin ninguna excepción él trabajaba con nosotros en depurar nuestra propia orquesta pero en la imaginación, para lo cual probaba nuestra entonación con su diapasón. La capacidad de cantar las notas con entonación perfecta era el medio que nos permitía desarrollar la habilidad de escuchar internamente una partitura sin la ayuda de grabación alguna. No nos permitía dirigir una orquesta en vivo hasta que la habilidad de escuchar una partitura internamente se había desarrollado completamente. Estoy profundamente agradecido y honrado de haber estudiado con él durante cinco años".

Juan Pablo Izquierdo también creó una experiencia de seminario única para un grupo de estudiantes seleccionado cada semestre. Estaba compuesto de intérpretes, compositores y directores, y tenía como propósito estudiar una sola obra en profundidad, en términos analíticos, históricos y musicales. La culminación de este estudio era la ejecución en concierto de la obra seleccionada. Entre las obras estudiadas y ejecutadas por los estudiantes figuraba el arreglo que Schoenberg hizo del Prélude a l'apres-midi d'un faune de Debussy, la Misa en Si menor de Bach y una velada con obras de John Cage. Estas experiencias de aprendizaje establecieron una vez más un estándar extremadamente alto de autopreparación e investigación, un rasgo característico de la obra profesional de Izquierdo.

A medida que la Carnegie Mellon Philharmonic se fortalecía, el maestro Izquierdo avizoró una oportunidad de vincular los dos medios en que participaba como director. Desde su posición como director musical de la Orquesta Claudio Arrau en Santiago, Izquierdo trabajó con numerosos músicos chilenos jóvenes. El maestro pensó que ellos podrían mejorar su nivel de aprendizaje mediante estudios profesionales en un conservatorio sobresaliente de los Estados Unidos. Inmediatamente se le vino a la mente la Carnegie Mellon School of Music. 
Después de obtener el compromiso de apoyo financiero de Igor Saavedra Gatica, Presidente de la Fundación Andes, Izquierdo desarrolló un programa de residencia en interpretación en el Carnegie Mellon, el que es similar a un programa de investigadores visitantes para jóvenes científicos. De acuerdo con sus propias palabras: "la idea era ampliar la experiencia orquestal de músicos de este nivel de calidad. Al mismo tiempo estos músicos acrecentarían nuestro programa orquestal y les proporcionarían a nuestros estudiantes la oportunidad de trabajar con intérpretes avanzados de otras naciones”. El programa se inició con seis músicos de Santiago cuidadosamente elegidos, quienes llegaron a Pittsburgh en enero de 1995. Este programa le abrió la puerta a más de 40 jóvenes profesionales de muchas otras naciones para la prosecución de estudios avanzados, además de Chile. Hasta el día de hoy el programa continúa acrecentando el entorno educacional de la Carnegie Mellon School of Music.

Durante la permanencia de Juan Pablo Izquierdo en el Carnegie Mellon, la Escuela de Música también lanzó una serie de transmisiones de radio denominadas International Music from Carnegie Mellon (Música internacional desde Carnegie Mellon), producido por WDUQ-FM, señal afiliada a la Radio Nacional Pública de Pittsburgh. Esta serie programó a intérpretes del cuerpo de profesores como el Carnegie Mellon Trio, Cuarteto Latinoamericano, Andrés Cárdenes y Julius Baker, artistas y compositores famosos invitados y la Orquesta Filarmónica de la escuela, bajo la dirección de Juan Pablo Izquierdo. Se transmitía en tres idiomas y tenía como animador a Ricardo Lorenz, un especialista en música latinoamericana con manejo fluido del inglés y del castellano. La serie constaba de seis partes y fue transmitida por muchos años en estaciones radiales públicas por los Estados Unidos además de México y Latinoamérica.

Hoy día su legado como director musical y batuta de la Carnegie Mellon Philharmonic perduran en un número de fonogramas que han sido entusiastamente aclamados y que los ha editado Mode Records. Estos fonogramas recogen la notable habilidad de Izquierdo en la ejecución de las obras de los maestros del siglo XX. Tres ejemplos particularmente notables son los siguientes: Xenakis/Varése, Mode Records 58 (1997); Giacinto Scelsi: The Orchestral Works, Mode Records 95 (2001) y George Crumb: Black Angels, Makrokosmos III, Mode Records 170 (2006). A continuación se presentan algunas de las críticas escritas acerca de estos fonogramas:

Iannis Xenakis

Dämmerschein/Persephassa/La Déesse Athéna

Mode 58

Harold Lewis afirma lo siguiente en una reseña publicada en internet (rec. music.classical.recordings):

"Mode 58, el más reciente CD con la serie de grabaciones de obras de Xenakis, contiene, además de las tres obras de Xenakis, la mejor interpretación y grabación de Amériques de Varèse. Juan Pablo Izquierdo dirige la excelente Carnegie Mellon Philharmonic en una lectura de formidable autoridad, completamente atenta a los cambios de carácter, movimiento y textura de la obra. Presenta los intrincados detalles y sutilezas de la partitura, junto a la fuerza cabal de la música. La interpretación se apoya en una soberbia labor de ingeniería, que permite un maravilloso ambiente de sala de concierto, con un 
rango amplio pero natural de dinámica y sonido que está limpia y claramente enfocado... Los entusiastas por la música de Varèse que todavía no conocen esta grabación deberían conseguir un ejemplar lo antes posible".

-Harold Lewis, reseña internet

Desde París, Francia:

“...una nueva grabación de la obra clásica para percusión Persephassa, compuesta el año 1969, que ha sido largamente esperada. En lugar de completar el disco con otras obras de Xenakis para orquesta, Juan Pablo Izquierdo eligió la obra clásica de Varèse Amériques escrita en 1921. Esto sugiere una comparación con la poderosa grabación de la Orquesta Filarmónica de Nueva York realizada por Boulez. De hecho, los estudiantes de Carnegie Mellon se equiparon bastante bien. La mezcla de sonido pone de relieve algunas curiosas secciones de actividad instrumental que yo no había notado anteriormente. El final deja a Boulez fuera del campo de juego, con la apocalíptica sirena barítono de incendio proveniente de la Compañía de Bomberos de Mount Lebanon-iQue Dios se apiade de nuestros oídos si alguna vez su casa se incendia en Pittsburgh!".

-Dan Warburton, Paris Transatlantic Review, noviembre, 2000

Finalmente, Ben Watson de The Wire escribe:

"La cantidad de eventos comprimidos en diez minutos es sorprendente. La interpretación, supervisada por el compositor, fue realizada por la Carnegie Mellon Philharmonic bajo la dirección de Juan Pablo Izquierdo mientras que la grabación corresponde a Ricardo Schulz. Ambas capturan la actividad múltiple de la partitura de Xenakis. La fuerza que se hace a la compostura clásica es inmensa. No obstante, el despliegue de recursos sinfónicos permite que Xenakis surja como su aspirante al legado clásico". "La obra Amériques de Edgard Varèse proviene del futurismo del Nuevo Mundo de 1922. En la estética histórico materialista de Varèse el gesto nunca va más allá de la realidad sonora. La interpretación aquí es veloz y la escritura de las cuerdas se realiza con una precisión minuciosa. Posiblemente el motivo inicial de la flauta se interprete de manera algo suave. No obstante, hacia el final Izquierdo se maneja para producir la coherencia por la vía de un cataclismo de acuerdo a la intención de Varèse. Media hora de excelente música nueva en discos excepcionales".

-Ben Watson, The Wire, marzo, 1998

\section{Giacinto Scelsi}

\section{The Orchestral Works Vol. 1}

\section{Carnegie Mellon Philharmonic}

\section{Juan Pablo Izquierdo}

Mode 95

"Este es un glorioso Scelsi-una novedad indispensable para los aficionados a la música de Scelsi, y el fonograma perfecto para convertir tanto a los escépticos como a los inocentes. Asumo mi apreciación de Scelsi de manera seria pero cuidadosa. Este es un disco que toco cuando no hay nadie en la casa".

"Este es el segundo de la serie de los volúmenes que Mode ha dedicado a Scelsi bajo el sugerente título The Orchestral Works I. Sirve como una perfecta introducción a la obra del compositor. La música orquestal es más imponente y madura que aquella que contiene la colección Accord. La combinación de pequeñas obras vocales con enormes piezas orquestales hace patente los intereses y preocupaciones de Scelsi por lo que no 
es occidental. Juan Pablo Izquierdo dirige la Carnegie Mellon Philharmonic con una mayor serenidad y profundo goce que es el que yo aprecio en su disco con música de Xenakis y Varèse [Mode 58] por lo que no puedo esperar por escuchar el resto. De verdad es más simple escuchar la música de Scelsi que describirla. De todo corazón recomiendo este disco".

-Grant C. Covell, La Folia, reseña online, agosto 2002 [www.lafolia.com]

"Otro rótulo en minúsculas para un CD que hace una contribución mayor a la música contemporánea. Mode presenta su primer volumen con las obras para orquesta de Giancinto Scelsi...este disco contiene una interpretación vívida en un sonido excelente de Hymnos, Hurqualia y Konx-Om-Pax. La mejor manera de describir las obras de gran tamaño de Scelsi es pensar en una fuerza irresistible que rueda en una escarpada colina, de manera cada vez más fuerte e insistente, y, claro, aplastando todo a medida que desciende. Amo esta música".

-Stephanie von Buchau, The Tribune, Oakland CA, 21 de febrero, 2001

\section{George Crumb}

\section{Black Angels and Makrokosmos III}

\section{Mode 170}

Este fonograma obtuvo el prestigioso premio Diapason d'or en Francia. Lo otorga la revista francesa Diapason que se dedica a las grabaciones de música clásica. En la reseña que escribiera para Diapason, Nicolas Baron se refiere a la grabación como "bien construida con una muy interesante espacialización de la fuente sonora tanto en anchura como en profundidad", a lo que agrega que es "muy transparente y con un hermoso ámbito dinámico".

Al ser notificado de este galardón, Juan Pablo Izquierdo comentó: "este premio confirma una vez más la gran calidad artística que la Universidad de Carnegie Mellon puede obtener con la estrecha colaboración de estudiantes, profesores y funcionarios para el beneficio de un gran compositor norteamericano".

Como una demostración adicional del legado del maestro Izquierdo se puede señalar lo siguiente:

$<$ Salabert, el editor de la obra de Xenakis, publicó una hermosa retrospectiva con una muestra en CD de su música. Muchas de las grabaciones de Juan Pablo Izquierdo se incluyeron el año 2001 en esta muestra.

$<$ Juan Pablo Izquierdo proporciona un análisis musical en la nueva edición de la partitura de orquesta de Hurqualia de Giacinto Scelsi (Ediciones Salabert, 2009).

$<$ Un capítulo del libro de Sharon Kanach, Performing Xenakis, "On Dammerschein", fue escrito por Riccardo Schulz y Juan Pablo Izquierdo como parte de Iannis Xenakis, Second Series [Pendragon Press, 25 de enero, 2010].

Probablemente la contribución más duradera que hizo Juan Pablo Izquierdo durante los dieciocho años en que se desempeñó como Director de Estudios Orquestales y Profesor de Música en la Universidad Carnegie Mellon se refleja en las vidas y carreras de cientos de músicos por todo el mundo, quienes tuvieron el privilegio de estudiar con él durante los años tempranos de su preparación preprofesional como intérpretes, compositores y directores de orquesta. 
Andrés Cladera, quien estudió dirección de orquesta con Juan Pablo Izquierdo, ha señalado lo siguiente acerca de sus estudios con Izquierdo:

"El maestro Juan Pablo Izquierdo ha sido una gran inspiración para mí tanto como director de orquesta y como persona. Su enseñanza moldeó mi integridad como director y cómo incorporarme a la música contemporánea con verdadera pasión.

Fue su amor por la música contemporánea que me inspiró la creación de la Microscopic Opera Company en Pittsburgh, un grupo que se concentra solamente en la ópera contemporánea de cámara escrita sobre textos en inglés. Maestro Izquierdo fue el catalizador para que yo llegara a ser el director de orquesta que he llegado a ser hoy día. Él me sigue guiando e inspirando mi compromiso de vida por la música”. 